\title{
Discoveries, not Inventions - Interview with Ernest Borisovich Vinberg
}

Alice Fialowski (University of Pécs and Eötvös Loránd University, Hungary), Joachim Hilgert (Paderborn University, Germany), Bent Ørsted (Aarhus University, Denmark) and Vladimir Salnikov (Luxembourg University, Luxembourg)

Ernest Borisovich Vinberg received the Distinguished Speaker Award in 2016 from the European Mathematical Society. He delivered his talk at the 50th Sophus Lie Seminar in Bedlewo, Poland. On this occasion, we asked him to give an interview for the EMS Newsletter. The interview took place in Będlewo, Poland, on 27 September 2016.

Professor Vinberg, we are very happy that you came here to this 50th Seminar "Sophus Lie" and we would like to ask a few questions.

To begin with, who got you into your research?

In fact, I had two advisors: Evgeny Borisovich Dynkin and Ilya Iosifovich Piatetski-Shapiro. They were both distinguished mathematicians. Dynkin was a brilliant lecturer, attracting a lot of young people, but PiatetskiShapiro posed the problem which was kind of a challenge. This problem concerned homogeneous bounded domains. The question posed by Élie Cartan was whether any such domain is symmetric, and Piatetski-Shapiro gave a counterexample in dimension 4 . Then a problem arose to give a classification of homogeneous bounded domains in complex spaces. And this turned out to be related to classification of homogeneous convex cones in real vector spaces, which was the subject of my $\mathrm{PhD}$ thesis. ${ }^{1}$

\section{And when was it?}

I entered the graduate school in 1959 but I began my scientific research some time earlier. My first published paper was my diploma work on invariant linear connections in homogeneous spaces, which was fulfilled under the guidance of Dynkin in the academic year 1958/59.

\section{Did you continue working with Dynkin and Piatetski-} Shapiro? Tell us something about your two advisors.

I never really worked with Dynkin because he had completely switched to probability theory by that time. I continued to work on homogeneous Kähler manifolds with Piatetski-Shapiro and another of his students, Simon Gindikin, up to 1965. After that, I turned to other problems. Some of them were influenced by my previous work, some of them not, but I became more or less independent at the age of about 27. But the topic of my

1 Кандидатская диссертация in Russian.

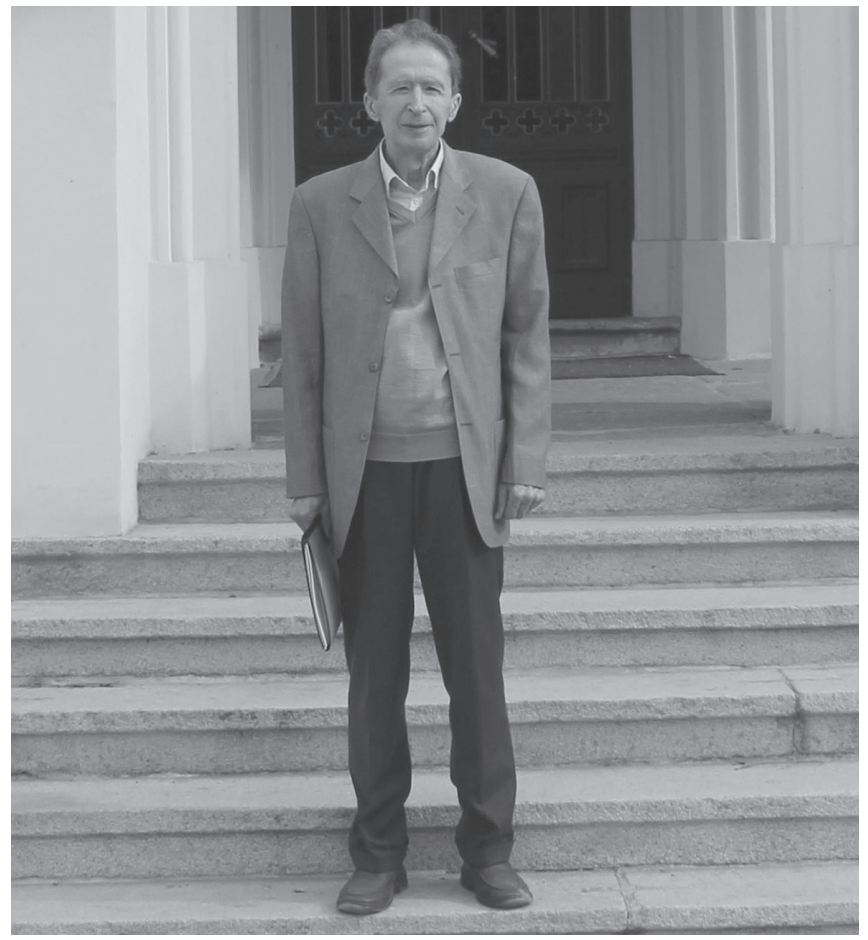

Ernest Borisovich Vinberg, Będlewo 2016. (All photos in this article by Janusz Grabowski.)

doctoral thesis ${ }^{2}$ was also related to Piatetski-Shapiro's question. It concerned the Selberg conjecture about arithmeticity of discrete subgroups, so-called lattices, in semisimple Lie groups. Piatetski-Shapiro was interested in this problem but he did not manage to solve it, although he had some results in this direction. I developed a theory of hyperbolic reflection groups, which permitted me to construct many counterexamples at rank one. After that, Margulis proved his famous theorem for higher rank, answering affirmatively the Selberg conjecture. My work on hyperbolic reflection groups had two sources: one was Dynkin's theory of simple roots, which is tightly related to finite reflection groups; and the other was the theory of automorphic forms for lattices in semisimple Lie groups, which was a favourite subject of PiatetskiShapiro. So in a sense I continued studying some questions they posed to me (both Dynkin and PiatetskiShapiro). In 1966, there was the International Congress

2 Докторская диссертация in Russian - roughly equivalent to habilitation in some countries. 


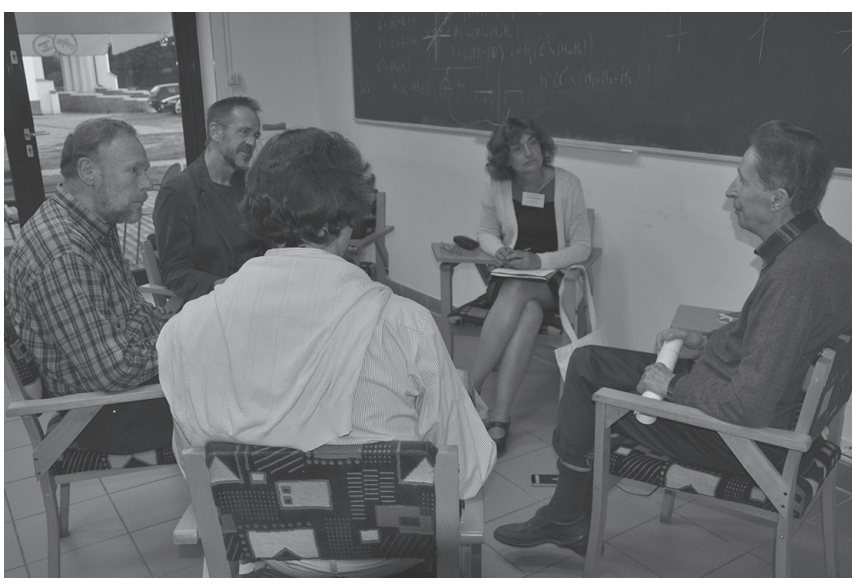

Interviewing Ernest Borisovich.

of Mathematicians in Moscow and I gave two talks there. One of them was related to our common activity with Piatetski-Shapiro and Gindikin, and another was about hyperbolic reflection groups.

The Moscow school at that time was really famous. Who were your colleagues from the same generation?

Yes, it was a really remarkable time; some people call it the Golden Age of Moscow Mathematics. And especially our course was very strong. Many people from our course were hired to teach at our mathematics faculty in 1961. It was an initiative of academician Kolmogorov. Among them, apart from me, there were Kirillov, Arnold, probability theorists Shur and Tutubalin, topologists Arkhangelski, Pasynkov and Ponomarev, and some others. I was very happy that this happened.

How do you choose a problem to work on? I mean, how do you decide if the problem you think of is worth your time? You are known as having solved many different problems; you are not a researcher who goes in one direction only. What do you need to like in a problem to get started?

Yes, I worked on different problems but they were somehow related. All the areas of mathematics are related. It is difficult for me to say how I choose a problem. If something is interesting for me and I feel that I can do something in this direction, I try to do it. If it is not interesting for me, I don't try to do it.

Can you give something like a criterion of what makes it interesting for you?

I think there are two types of mathematical results: inventions and discoveries. I realise the importance of inventions but I prefer discoveries. Sometimes, when I obtain a result, I have a strong feeling that I am discovering something existing in nature. And I don't have such a feeling with respect to some other works and mathematical results; they seem to be more inventions - creations of the human brain - rather than something really existing in nature.

What is your favourite discovery?

I hope I have not made it yet.
If you have to choose among those you have discovered already?

I think these are, first, the theory of hyperbolic reflection groups, second, my development of invariant theory, which I call the "effective invariant theory" (related to graded Lie algebras) and maybe also my work about invariant orderings on semisimple Lie groups. Quite recently I began to study the so-called Chevalley-type theorems. There is a famous theorem of Shephard-ToddChevalley about the criterion for the algebra of invariants of a finite linear group to be free. It says that it is exactly the case when the group is generated by complex reflections. The same question can be posed for infinite reflection groups and the natural setting is to study complex reflection groups in symmetric domains, namely in complex balls and so-called future tubes, which are the only symmetric domains admitting reflections. Recently I obtained some results in this direction, together with Osip Schwarzman, my former student. Maybe it will be my favourite result for the moment.

You've conducted a very famous seminar for several decades, together with Onishchik, and produced a book out of it. Can you tell us more about this seminar?

Yes. It was, in fact, a continuation of the famous seminar ran by Dynkin - our common teacher - after he had switched to probability theory. He was a student of Kolmogorov - a famous probabilist and the creator of axiomatic probability theory. And he started with some work in probability theory. But after that, he attended a seminar of Gelfand, and Gelfand suggested he prepare a talk on the classification of simple Lie algebras. Preparing this talk, Dynkin discovered his famous simple roots. At that time, he was a student in the fourth year (it was during the war in 1944). After that, Dynkin got interested in the theory of simple Lie algebras and produced his famous works, which made him a classic in Lie theory. About 1955, he switched to probability theory and eventually became a classic in this theory, too. By the way, it is interesting that several years before his death in 2014, he did some joint work with my student Andrei Minchenko on simple Lie algebras.

Coming back to your question, our seminar started in 1961, when we were both young teachers in our department. First, we tried to study all mathematics, starting from Cantor's set theory. But soon we realised that we had been quite naive and concentrated on Lie group theory. At that time, the famous Chevalley seminar was available and the Chevalley monograph "Theory of Lie groups" appeared. We understood that we should study algebraic groups in connection with Lie groups.

There were many enthusiastic young people attending our seminar. Everything was going on in the following way: we (me and Onishchik) presented some theory, mostly in the form of a series of problems. All the participants - there were about 25 of them - split into several groups, discussing the problems and the solutions between two sessions of the seminar, and the most interesting solutions were presented at the next session. Then we continued presenting some new theory. 
As a result of this study, our book Seminar on Lie Groups and Algebraic Groups appeared (in the English translation, "Seminar" was omitted). First, it was prepared with the help of some participants of our seminar, who wrote down what we told them and the problems, and so on. Then me and Onishchik rewrote all this, edited it, and in this way our book appeared. It retains the style of the seminar. The theory is presented in a series of problems, which the reader is supposed to try to solve on his/her own, but there are some hints at the end of each chapter. There are also some exercises. This book was used by several generations of students and graduate students of our department.

After that, we switched to invariant theory. We studied it together, the leaders and the participants. As a result, some of us became experts in this theory and made some contributions. During this period, V. Popov joined me and Onishchik as a leader of the seminar.

Apart from these, many other topics were also presented at our seminar. One year we studied supermathematics. Perhaps you know that one of the founders of supermathematics was Berezin, who was our elder scientific relative, a student of Gelfand. And also some other topics appeared, e.g. discrete groups and applications of Lie theory in mathematical physics. Dmitry Alekseevsky, one of the participants of our seminar, understood mathemetical physics very well and he presented a series of talks.

The seminar lasted in this way for about 50 years. But these days, Onishchik is not able to help with this anymore and there are new leaders: my younger students (nowadays colleagues) Timashev and Arzhantsev. Unfortunately, this is going on in a much worse way because fewer students come to study mathematics. The younger generation is less motivated in this and this is quite sad...

You were the founder of the Journal "Transformation Groups". Could you tell us about the first steps?

We founded this journal together with my former student Vladimir Popov, with active participation and help of Ann Kostant, the mathematics editor of Birkhäuser at that time, and have been running it (I hope, successfully) for 20 years. The first managing editors were (apart from me and Popov) C. De Concini, G. Margulis, A. Onishchik, G. Schwarz and M. Vergne. The whole editorial board consists of more than 30 mathematicians and is gradually being renovated. At different times, the managing editors have been M. Brion, P. Etingof, E. Frenkel, V. Ginzburg, W. Goldman, M. Kapovich, A. Kleshchev, I. Mirkovic, H. Nakajima, A. Premet and A. Zelevinsky. We reject more than half the submitted papers.

As was written in the preface to the first issue, the concept of a transformation group reflects the symmetry of the world, which is perceivable so far as it is symmetric (but we do not know why it is so remarkably symmetric). All my personal work is related to different kinds of transformation groups.

I first knew you as a teacher-during my first years at the Mathematics Department in Moscow. So my ques-

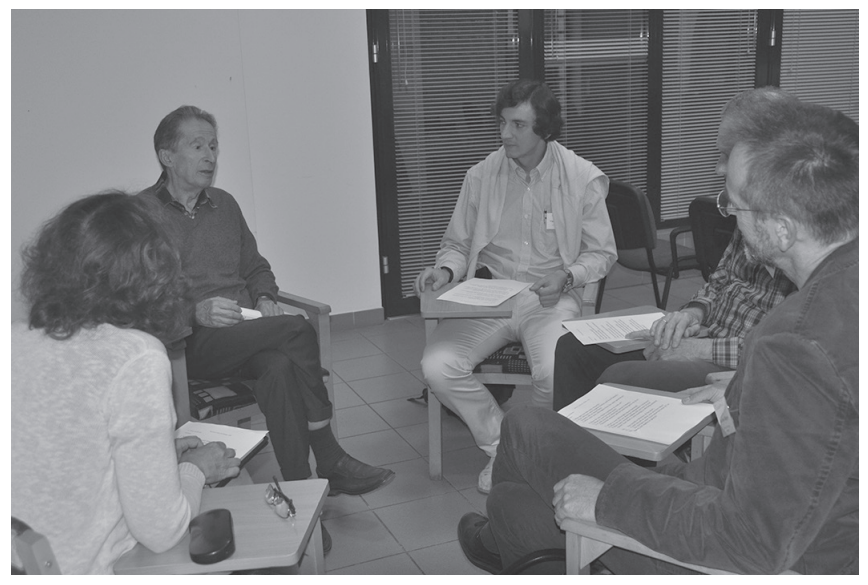

During the interview.

tion is: do you have any teaching philosophy, any principles that you would like to share?

My first principle is that it is not so important what to teach but the most important thing is how to teach. Because it is clear that most of the theorems that we teach to our students will never be needed for them when they graduate from university. But we have to teach them the right thinking. The second principle is that one should try to avoid tedious calculations, replacing them with the ideas that permit getting the same result without calculations.

And Mechmat ${ }^{3}$ is not the only place where you can learn mathematics in Moscow.

It used to be essentially the only place. But then the Independent University appeared and, in recent years, the Faculty of Mathematics of the Higher School of Economics has appeared.

\section{Would you like to say something about the Independent University?}

The Independent University was a very important and useful project. But I would like to say that it is not really independent. I know only one person (Valentina Kirichenko) who graduated from the Independent University and did not study at the Maths Faculty of Moscow State University. The Independent University is rather a system of advanced courses: they do not teach elementary algorithms. But I think it was very important for several generations of young people and also for talented mathematicians who could not go to teach at Moscow State University for some reason.

You have supervised a lot of students: more than 40 PhD theses and several habilitations. Do you have any strategy? What is your approach to advising students? Well, I have no special strategy. I just try to make them get interested in mathematics. I try to find some interesting problems, which they can solve. But I'm afraid I don't pay enough attention to my students.

3 Faculty of Mechanics and Mathematics of MSU. 
You have been an excellent teacher and an outstanding researcher for all your life. And you are one of the people who stayed in Moscow...

Yes, I never considered the possibility of emigrating. I stayed in Moscow. But over the last 20 years, I have visited Germany, namely Bielefeld University, every Summer, for two to three months. They initiated this, nominating me for the Alexander von Humboldt Prize, which I won in 1997. After that, they continued inviting me in the framework of their SFB (Sonderforschungsbereich). By the way, this university has successfully nominated many Russian mathematicians for the Humboldt prize: Alexander Merkurjev, Sergei Adjan, Vladimir Platonov and others. In Bielefeld, I collaborated with local mathematicians J. Mennicke, H. Helling and H. Abels, and also with other guests from all over the world. Visiting Bielefeld University is sort of my second life. Being in Moscow, I am always quite busy with many things; many people disturb me and want something from me. And when I go to Bielefeld, I relax and reflect on problems that I am interested in, I talk to my colleagues and so on. So my life is divided into two different parts, each of them being very important for me.

You told us before that right now you are working on complex reflection groups. Could you share some other things that you currently find interesting, that you are working on at the moment? Some projects perhaps? Your current activities?

I am always working simultaneously on two to three topics. For the moment, I am working on complex reflection groups as you said and on some "non-abelian gradings" of simple Lie algebras, on which I am going to talk tomorrow at this conference. I am also reflecting about some problems of equivariant symplectic geometry, continuing my previous results.

And we've heard that you are also involved right now in teaching as always. What classes do you teach?

The course of algebra for the second year undergraduate students and the advanced course in invariant theory for students starting from their third year. I am also running two seminars: one research seminar we have already discussed and another one for students, which is called "Algebra and Geometry". At the latter one, we try to show relations between algebra and geometry. For example, for several years we studied relations between algebraic invariant theory and the theory of automorphic forms. Namely, due to Torelli type theorems, automorphic forms can be studied by means of geometric invariant theory, realising arithmetic quotients of symmetric domains as the moduli spaces of some classes of algebraic varieties.

And nowadays, how many students attend those seminars, since you said there are fewer interested students? Yes. Unfortunately, only a few students attend our research seminar in Lie groups and only 10-12 students attend our seminar "Algebra and Geometry". But, from time to time, I still have talented students coming to me,

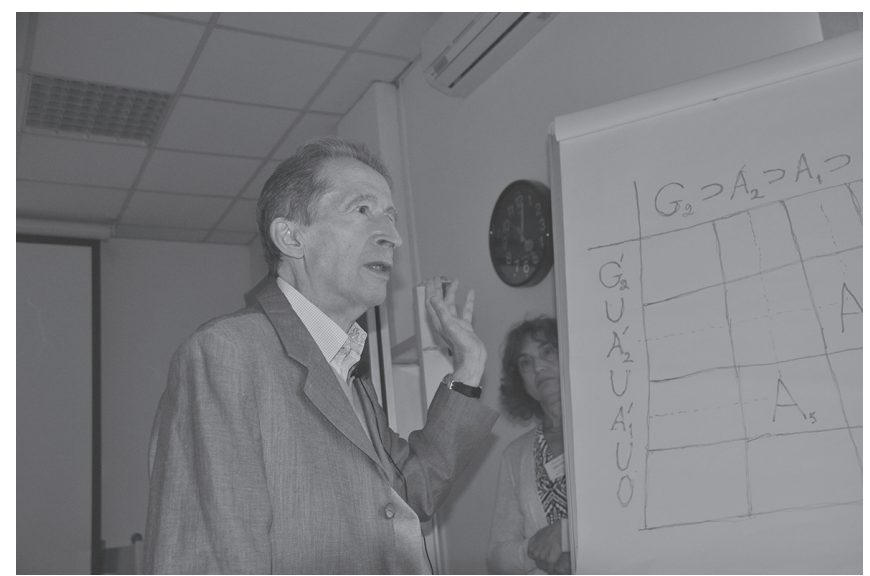

E.B.Vinberg delivers his EMS lecture.

who are interested in doing mathematics and not business, and some of them are not even going to emigrate.

\section{Thank you very much!}

Thank you for your interest!

P.S. Off the record, Ernest Borisovich told us about two of his interviews with Dynkin in the USA in 1992 and 1999. A huge collection of interviews of Dynkin with many Russian and Western mathematicians who came to visit him in the United States, is now available at the online library of the Cornell University: http://dynkincollection.library.cornell.edu/.

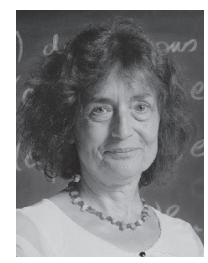

Alice Fialowski is professor of mathematics at the Institute of Mathematics, University of Pécs, and at the Eötvös Loránd University in Budapest, Hungary. Her research interests are Lie theory, cohomology, representation and deformation theory, with applications in mathematical physics.

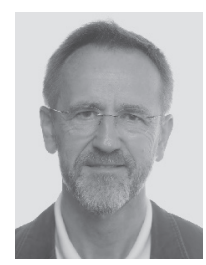

Joachim Hilgert is professor of mathematics at the Institut für Mathematik at Paderborn University, Germany. His research interests are harmonic analysis, representations of Lie groups, symplectic geometry, and supermanifolds.

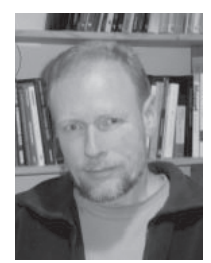

Bent $\emptyset$ rsted is professor of mathematics at the Department of Mathematics, Aarhus University, Denmark. His research interests are harmonic analysis, representations of Lie groups, conformal geometry, spectral geometry.

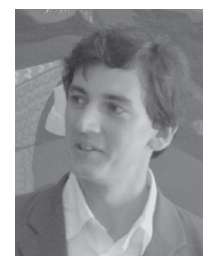

Vladimir Salnikov is a senior researcher in mathematics at the RMATH, University of Luxembourg, Luxembourg. His research interests are graded and generalized geometry, dynamical systems and integrability, applications to theoretical physics and mechanics. 